\title{
MANAGEMENT OF DISTAL HUMERUS FRACTURES WITH JESS FIXATOR
}

\author{
Prakriti Raj Kandel $^{1}$, Kishor Man Shrestha ${ }^{1,}$ Laxmi Pathak $^{2}$
}

\section{ABSTRACT}

INTRODUCTION: Fractures of distal humerus are rare comprising approximately $2 \%$ of all fractures and a third of all humerus fractures. Even with the development of newer fixation techniques, the treatment of distal humerus fractures remains a great challenge to any orthopaedic surgeon. Thus this present study was conducted to evaluate the results of Joshi's External Stabilization System (JESS) in the management of distal humerus fracture with or without intercondylar extension.

MATERIALS AND METHODS: This retrospective study was conducted by collecting records of thirty two adult patients who sustained distal humerus fracture and were managed with JESS fixation under anaesthesia over a period of two years in Universal College of Medical Sciences Teaching Hospital (UCMSTH) after obtaining permission from Institutional ethical committee.

RESULTS: According to AO (Arbeitsgemeinschaft für Osteosynthesefragen) classification, 2 patients had C1 fracture and 30 patients had C2 fractures. The mean duration of JESS application was 16 weeks. The mean follow up was 8 months. The functional outcome was evaluated by using Cassebaum's functional rating system. Among $\mathrm{C} 1$ fractures, $50 \%$ showed fair and $50 \%$ showed good result whereas among $\mathrm{C} 2$ fractures, $40 \%$ showed good, $46.66 \%$ showed fair and $13.33 \%$ showed poor results.

CONCLUSIONS: JESS fixation technique represents a viable option in the management of open as well as close intercondylar fractures of the distal humerus.

KEYWORDS: JESS; Intercondylar fracture; External fixation

1 Assistant Professor, Department of Orthopaedics and Trauma Surgery, Universal College of Medical Sciences \& Teaching Hospital, Bhairahawa, Nepal

2 Associate Professor, Department of Anaesthesiology, Universal College of Medical Sciences \& Teaching Hospital, Bhairahawa, Nepal

\author{
For correspondence: \\ Dr. Prakriti Raj Kandel \\ Assistant Professor \\ Department of Orthopaedics, \\ Universal College of Medical Sciences \&Teaching \\ Hospital, Bhairahawa, Nepal \\ E-mail:pratul22@gmail.com
}




\section{INTRODUCTION}

Fractures of distal humerus are rare comprising approximately $2 \%$ of all fractures and a third of all humerus fractures. Even with the development of newer fixation techniques, the treatment of distal humerus fractures remains a great challenge to any orthopaedic surgeon. Open reduction internal fixation with plating is well accepted as the standard treatment for these fractures as it confers good anatomical reduction and early mobilization of the joint. However, this technique may be associated with inferior postoperative results and a higher risk of infection in both open as well as close fractures. ${ }^{1-5}$ External fixation with JESS appears to be an alternative option in such cases because better anatomical reduction can be achieved with minimal damage to soft tissues. Later on, removal of this fixator is also easier.

The present study was conducted to evaluate the results of JESS fixator in the management of distal humerus fracture with or without intercondylar extension in terms of clinical and radiological outcome.

Adult patients with American Society of Anaesthesiologist (ASA) Physical Status 1 and 2 with both open and closed intercondylar fracture of distal humerus were included.

Patient aged less than 18 years or more than 70 years old, associated other injury or concomitant limb injury, associated neurovascular deficit, serious medical or surgical illness with ASA more than 2 and old fractures of more than 3 weeks were excluded.

\section{PROCEDURES}

This retrospective study was conducted in UCMSTH from the records of patients underwent JESS fixation for distal humerus fracture after taking permission from Institutional ethical committee over a period of two years from Jan 2013 to Dec 2015.

Initial radiographs included were antero-poserior (AP) and lateral views of the elbow [Fig.1]. If open fracture the extent of the wound and its contamination was assessed. Distal neurovascular status was checked before attempting any surgical intervention. After proper clinical examination and necessary laboratory investigations, pre-anaesthetic evaluation for the fitness to deliver anaesthesia was carried out and informed written consent was taken one day prior to the schedule surgery date. Anesthesia techniques performed were General Anesthesia and Supraclavicular Brachial Plexus Block along with sedation. Monitoring of the vitals including postoperative pain of the patients was taken care by the anaesthesia team. All patients were operated within 3 days of hospital admission. Treatment outcome was evaluated as per Cassebaum's functional rating system. [Table 1] ${ }^{3}$
Table 1: Cassebaum's classification for elbow range of movement

\begin{tabular}{ll}
\hline Excellent & Flexion $>130$ and extension deficit $<15$ \\
Good & Flexion $>120$ and extension deficit $<40$ \\
Fair & Flexion $>110$ with any extension deficit \\
Poor & Flexion $<110$ \\
\hline
\end{tabular}

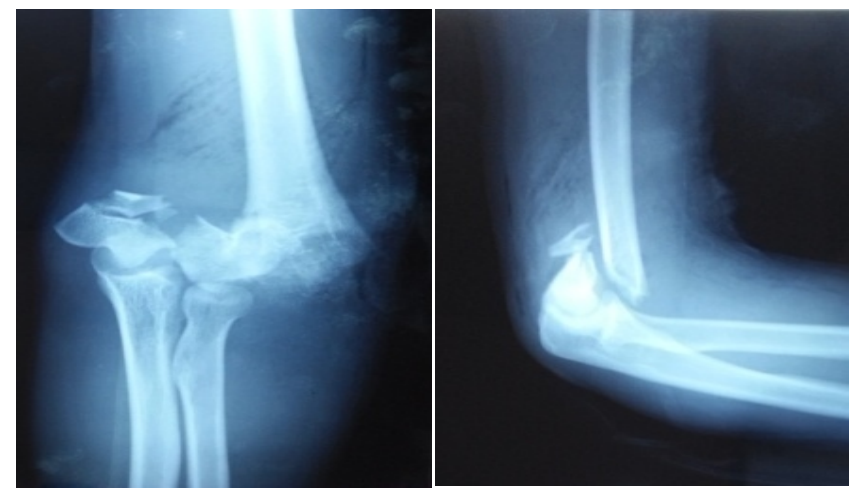

Fig. 1 Preoperative X-ray AP and Lateral views

JESS is a simple, light, highly modular mini external fixator system which systematically addresses a wide range of complex problems in the management of forearm and hand, invented by Dr. B. B. Joshi from Bombay. This system has high safety profile and unparalleled ease of application. It can be applied easily by any surgeons even in the remote areas with minimum instrumentation. It provides a simpler alternative to the presently available modalities of the treatment. It allows minimum invasive techniques.

The construction of JESS frame was planned. The components of the JESS included Kirschner wires (K-wires) of 2.0 and $2.5 \mathrm{~mm}$ thickness, Link joints $3 \times 3$ size with two offset holes to which K-wires and connecting rods are clamped, Connecting rods of 3 to $4 \mathrm{~mm}$ in diameter and of suitable lengths, Allen Wrenches to tighten the link joints to Kwires and connecting rods and the wire cutter and benders to adjust the frame.

After delivering anesthesia, patient was placed in the lateral decubitus position. The arm was held outright and the elbow supported on a well-padded attachment to the operating table. A high, upper arm tourniquet was used with pressure not exceeding $250 \mathrm{~mm} \mathrm{Hg}$ for a maximum duration of 90 minutes. A midline skin incision was made on the posterior aspect of the distal arm, curving medially around the tip of the olecranon and extending to the subcutaneous border of the proximal ulna. The incision was carried to the triceps retinaculum and deep medial and lateral soft tissue flaps been raised. The ulnar nerve was identified proximally and tagged with a penrose drain or vessel loop. The Triceps muscle was splitted in the 
line of fibers and the fracture was exposed. Thorough debridement with excision of devitalized soft tissue was carried out. Reduction of the intercondylar region was achieved primarily after satisfactory exposure and careful isolation of ulnar nerve was done. Care was taken not to denude the bone fragments of the soft tissue attachments as the $\mathrm{K}$-wire needs minimal purchase area. The intercondylar fragments were reduced under compression with a bone clamp and fixed with a $\mathrm{K}$-wire from medial to the lateral side. Subsequently, the intercondylar fragment consisting of the medial and lateral condyle was reduced to the metaphysis. Two crossed K-wires were passed obliquely upward, one from each condyle crossing the fracture site, taking purchase in the opposite cortices proximal to the facture. Once fixation was secured, the elbow was checked for range of motion (ROM) and stability of the construct. Finally, 2 K-wires were introduced proximal to the fracture site in the distal humerus in the perpendicular direction to the shaft of the humerus through skin with soft tissue dissection under direct vision to avoid radial nerve injury. The wires were then advanced through the medial soft tissues without injuring the isolated ulnar nerve. These K-wires were kept throughout the skin on either side. All wires inserted through the skin, and special attention was made to relax the skin every time a $\mathrm{K}$-wire was passed to prevent tension during wound closure. Oblique K-wires, reconstructing the pillars, were bent just outside the skin surface to make them parallel to the rest of the wires. All Kwires were cut outside the skin surface at the optimum length $(3-4 \mathrm{~cm})$ so that they could be attached to the connecting rods ( 2 on either side) with the help of link joints [Fig.2], [Fig.3].

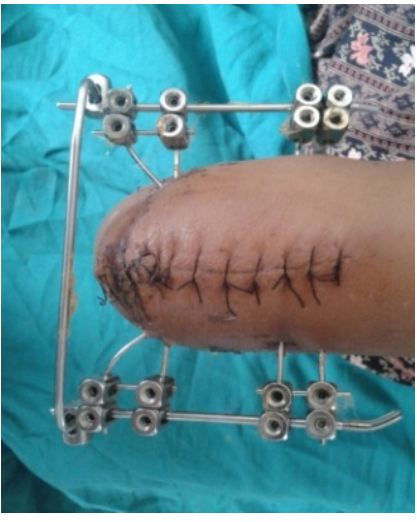

Fig.2 JESS in situ postoperatively

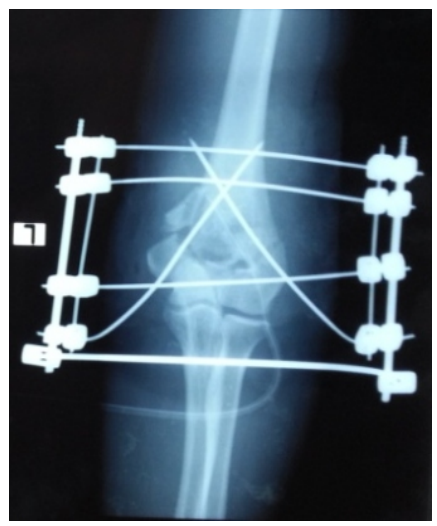

Fig.3 Immediate Postoperative $X$ ray
Thus, a bilateral uniplanar external fixator assembly was created. Surgical wounds were closed in a routine fashion over a drain and a dressing was applied. The limb was kept elevated in the immediate postoperative period. The drain was removed after 24 hours. Intravenous antibiotics were administered for 48 hours postoperatively. Active and passive elbow motion was initiated on postoperative day 3 to 5 after subsidence of fracture swelling or blister, which was feasible in all cases as the limb was elevated and immobilized in extension for the initial postoperative period. Suture removal was carried out on the fourteenth day postoperatively.

Pin tract dressing was done with antiseptics in every alternate day and Patients were followed up at 2 weeks, 4 to 6 weeks, 10 to 12 weeks, 6months, and 1 year intervals after surgery [Fig.4], [Fig.5], [Fig.6], [Fig.7] and [Fig.8]. The minimum follow-up duration was 3 months. Radiographs were taken at 6 and 12 weeks postoperatively.

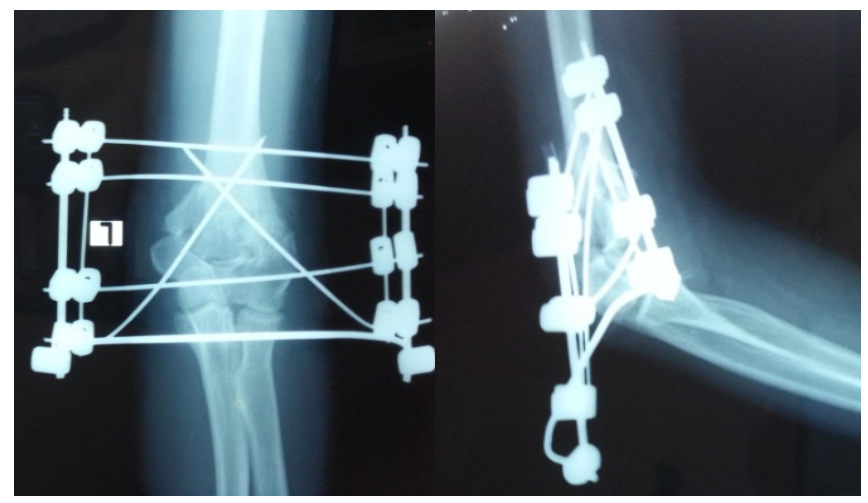

Fig.4: AP and Lateral X ray at $6^{\text {th }}$ Postoperative week

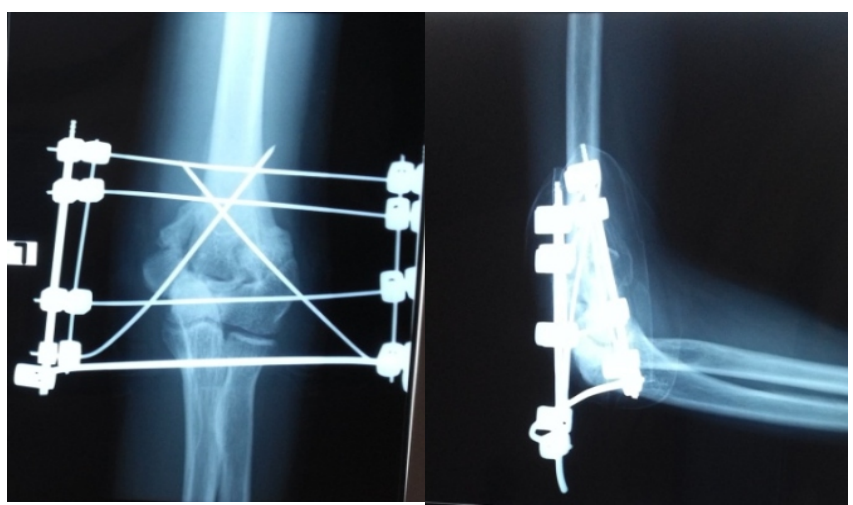

Fig.5: AP and Lateral $X$ ray at $12^{\text {th }}$ Postoperative week

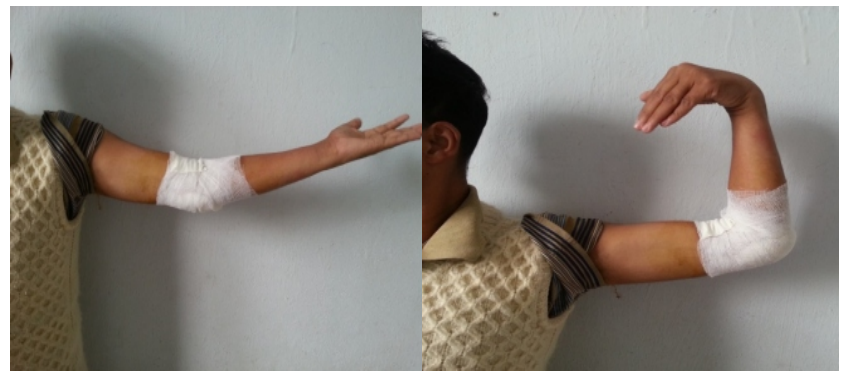

Fig.6: Range of movement at $12^{\text {th }}$ postoperative week (Immediately after JESS removal) 


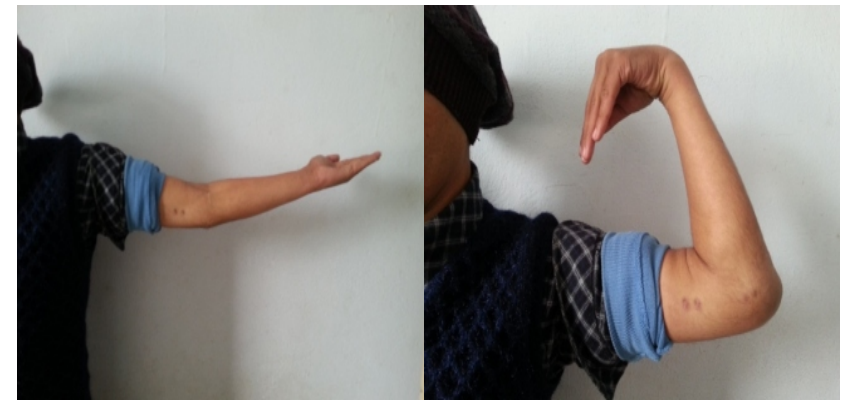

Fig.7: Range of motion at $6^{\text {th }}$ postoperative month

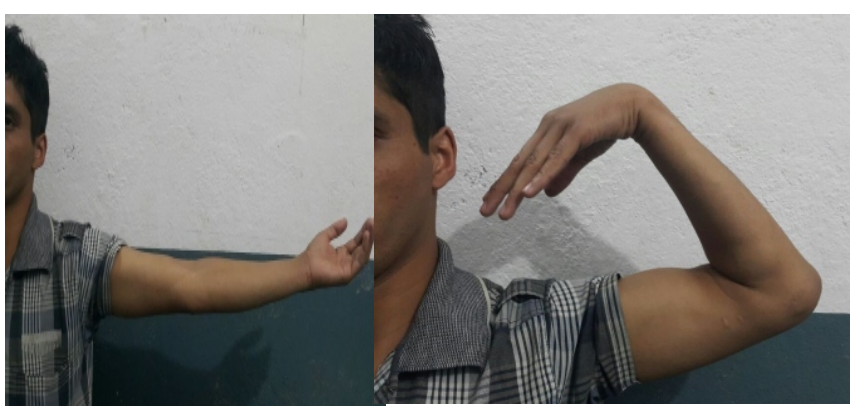

Fig.8: Range of motion at $12^{\text {th }}$ postoperative month

\section{RESULTS}

Records of total thirty two patients were studied. Among these, according to AO classification, two patients were $\mathrm{C} 1$ fracture and thirty patients were $\mathrm{C} 2$ fracture. The mean duration of JESS application was 16 weeks. The mean follow up was 8 months (3-18 months). The average range of movement was 20 to 120 degrees. 30 intercondylar fractures united, 2 cases went into delayed union at lateral column of supracondylar region, but the intercondylar fragments united well. 2 patients showed delayed union. Delayed union was defined as a fracture with little or inadequate evidence of healing even after 3 months. The fixator was removed in these patients due to pin tract infection without any change in rehabilitation protocol and managed with oral antibiotics and daily dressings. Rest of the patient showed radiologic and clinical evidence of union at 12 to 20 weeks (mean 16 weeks). The fixator assembly was removed after radiological evidence of union was found. There were no instances of nerve palsies or heterotrophic bone formation.

The ultimate functional outcome was evaluated by use of Cassebaum's functional rating system. $\mathrm{C} 1$ fractures showed fair to good results and $\mathrm{C} 2$ fractures showed poor to good results. Among $\mathrm{C} 1$ fractures 1 patient $(50 \%)$ showed fair result and another patient $(50 \%)$ showed good result, whereas among C2 fractures 12 patients (40\%) showed good result, 14 patients $(46.66 \%)$ showed fair result and 4 patients $(13.33 \%)$ showed poor result.

\section{DISCUSSION}

The complex articular anatomy of the distal humerus and limited bone stock for internal fixation welcomes continuous challenges, even to the most experienced surgeon. Substantial damage to the distal humerus usually results in some limitation of motion, pain, weakness and sometimes instability. The surgical goals of fixation of distal humerus intercondylar fractures are to reconstruct the articular surface and to fix the distal humeral articular complex to the medial and lateral columns. It has been noted that stable fixation and appropriate postoperative rehabilitation yield good to excellent results in $75 \%$ to $80 \%$ of patients. ${ }^{1,2,4,5}$

In the present study bilateral uniplanar construct was used to impart rigidity to the construct and permit early mobilization without compromising fracture stability. Thick K-wires of 2.0 $\mathrm{mm} 2.5 \mathrm{~mm}$ were used, depending on the thickness of the distal humerus and their respective cortices. All K-wires were introduced under direct vision after blunt dissection to avoid nerve damage. All patients with open injury were treated with the external stabilizing system only after thorough debridement and lavage with normal saline. JESS fixator offers the advantage of component removal in any clinic without using anesthesia in comparison to the possible necessity of hardware removal with ORIF, which requires anesthesia and extensive dissection and sometimes may lead to additional problems associated with a second surgical procedure. JESS fixation technique is a segmental fixation with a simple construct, thus technically less demanding with a shorter learning curve and can be mastered by a relatively junior consultant.

Safoury YA in $2011^{4}$ treated eight patients for post-infection nonunion of the supracondylar area of the humerus with the Ilizarov method and followed up for 3 years. All had undergone at least 2 previous failed operations. The patients were evaluated radiologically and clinically with an outcome survey using the Disabilities of the Arm, Shoulder and Hand (DASH) score. Solid union was achieved in all patients. None had recurrence of infection. Ilizarov treatment for postinfection nonunion of the supracondylar humerus was shown to be effective, reliable and tolerated by the patients.

Ilizarov method may be cumbersome and not acceptable to some patients. It may be kept in reserve as a last resort for chronic, unyielding fractures or infected cases rather than for primary management of open intercondylar fractures of the distal humerus. The JESS fixator frame in the form of a bilateral uniplanar construct with thick K-wires, as used in our patients, appears to be good construct.

Our study showed 2 patients with pin tract infection and 6 patients with joint stiffness. Records did not mention the complication like heterotrophic bone formation and nerve 
palsy, though these are common in various studies on management of intercondylar fracture humerus by open reduction internal fixation with plates. ${ }^{3,4,6-8}$

One hundred and eighty-four patients of intra-articular fractures of distal humerus were operated by posterior transolecranon approach by Babhulkar S et al. in 2011. Initially, in the first part, Chevron intra-articular osteotomy was done, later extra-articular olecranon osteotomy was routinely performed. Both columns were stably fixed by orthogonal methods. In 10 patients with severe comminution with bone loss, stabilization was achieved by parallel plating. The osteotomy was routinely stabilized by tension band wiring with two parallel K-wires introduced up to the anterior ulnar cortex. The results were evaluated by the staging system of Caja $e t$ al. at a minimum follow-up of 2 years. The results were ranging from excellent to good in 78 patients $(86 \%)$. The high rate of union can be achieved in complex intra-articular fractures of distal humerus if the proper principles of stable fracture fixation are followed, like a posterior transolecranon approach, dual fixation of both columns and restoration of the continuity of articular surface. Internal fixation is very demanding technically and difficult to hold in osteoporotic bones with comminuted fractures., 4 Conservative management does not give good results in such cases. The JESS fixation is a definitive and simple method in management of these cases. The fixator system is less cumbersome to the patient because of the low profile of the components as it can be removed easily without any need of anesthesia. It is better to check unrestricted motion in elbow, to ensure stability is restored, and to introduce all $\mathrm{K}$-wires under direct vision intra-operatively. Pin tract infection and pin loosening can lead to loss of fixation, alter the course management and potentially affect the ultimate functional outcome of the procedure. Hence, the importance of meticulous care of pin tract cannot be overlooked.

\section{CONCLUSION}

JESS fixation technique represents a viable option in the management of intercondylar fractures of the distal humerus.

\section{REFERENCES}

1. Babhulkar S BS. Controversies in the management of intraarticular fractures of distal humerus in adults. Indian J Orthop. 2011; 45: 216-25

2. Galano GJ AC, Levine WN. Current treatment strategies for bicolumnar distal humerus fractures. J Am Acad Orthop Surg. 2010; 18: 20-30.

3. Qureshi KK SM, Yasin G. Assessment of elbow function after stable fixation of AO type $C$ fracture the distal humerus in adults. Professional MedJ2005; 12: 331-5.
4. Safoury YA AM. Treatment of of post-infection nonunion of the supracondylar humerus with Ilizarov external fixator. J Shoulder Elbow Surg 2011; 20: 873-9.

5. Zlotolow DNCL, Barron OA, Glickel SZ. Surgical exposure of the humerus. . JAm Acad Orthop Surg 2006; 14: 754-65.

6. Cheung EV SS. Surgical approaches to the elbow. J Am Acad Orthop Surg. 2009; 17: 325-33.

7. Sanchez-Sotelo J TM, O'Driscoll SW. Complex distal humeral fractures: Internal fixation with a principle-based parallel-plate. Surgical technique. JBone Joint Surg Am. 2008; 90: 31-46.

8. O'Driscoll SW S-SJ, Torchia ME. Management of the smashed distal humerus. Orthop Clin North Am. 2002; 33: 19-33. 